\title{
Nitrogen Metabolism in Sinorhizobium meliloti-Alfalfa Symbiosis: Dissecting the Role of GInD and PII Proteins
}

\author{
Svetlana N. Yurgel, ${ }^{1}$ Jennifer Rice, ${ }^{1}$ and Michael L. Kahn ${ }^{1,2}$ \\ ${ }^{1}$ Institute of Biological Chemistry and ${ }^{2}$ School of Molecular Biosciences, Washington State University, Pullman 99164-6340, \\ U.S.A.
}

Submitted 23 September 2011. Accepted 1 November 2011.

To contribute nitrogen for plant growth and establish an effective symbiosis with alfalfa, Sinorhizobium meliloti Rm1021 needs normal operation of the GInD protein, a bifunctional uridylyltransferase/uridylyl-cleavage enzyme that measures cellular nitrogen status and initiates a nitrogen stress response (NSR). However, the only two known targets of GInD modification in Rm1021, the PII proteins GInB and GInK, are not necessary for effectiveness. We introduced a $\mathbf{T y r} \rightarrow$ Phe variant of GInB, which cannot be uridylylated, into a $g \ln B g \ln K$ background to approximate the expected state in a $g \ln D$-sm2 mutant, and this strain was effective. These results suggested that unmodified PII does not inhibit effectiveness. We also generated a $g \ln B g \ln K$ $g \ln D$ triple mutant and used this and other mutants to dissect the role of these proteins in regulating the freeliving NSR and nitrogen metabolism in symbiosis. The $g \ln D$ $\mathrm{sm} 2$ mutation was dominant to the $g \ln B g \ln K$ mutations in symbiosis but recessive in some free-living phenotypes. The data show that the GInD protein has a role in free-living growth and in symbiotic nitrogen exchange that does not depend on the PII proteins, suggesting that $S$. meliloti GInD can communicate with the cell by alternate mechanisms.

The bacterial nitrogen stress response (NSR) circuit (Fig. 1) plays an important role in regulating nitrogen exchange in the Sinorhizobium meliloti-alfalfa symbiosis (Yurgel and Kahn 2008). As in many other bacteria, the core elements of the $S$. meliloti Rm1021 NSR regulation cascade include a bifunctional uridylyltransferase/uridylyl-cleavage enzyme, encoded by the $g \ln D$ gene, and two PII proteins, GlnB and GlnK (Arcondéguy et al. 2001; Yurgel and Kahn 2008; Yurgel et al. 2010). In nitrogen-starved cells, when the concentration of $\alpha$ ketoglutarate is high, GlnD uridylylates the PII proteins which, in turn, activate the bacterial NSR, leading to more efficient ammonia assimilation and enabling the induction of nitrogenscavenging pathways. In the presence of a good nitrogen source, these responses are not needed and the high glutamine concentration stimulates GlnD-dependent deuridylylation of the PII proteins (Arcondéguy et al. 2001).

$S$. meliloti Rm1021 NSR regulation shares some similarities with NSR in enteric bacteria. For example, under high nitrogen conditions, GlnB interacts with NtrB, the sensor component of the NtrB/NtrC system, promoting dephosphorylation of the $\mathrm{NtrC}$ response regulator and inhibiting activation of operons

Corresponding author: S. N. Yurgel; E-mail: syurgel@wsu.edu; Telephone: +1.509.335.8617; Fax: +1.509.335.7643. involved in ammonium assimilation and nitrogen metabolism (Atkinson and Ninfa 1998; Yurgel et al. 2010). In free-living cells of $S$. meliloti, GlnB and GlnK can partially substitute for each other as regulators of the NSR. Deletion of either $g \ln B$ or $g \ln K$ resulted in partial inhibition of glutamine synthetase I (GSI) by adenylylation in cells grown under nitrogen limitation and, in the absence of both GlnB and GlnK, GSI was highly adenylylated under conditions where it would normally be unmodified (Yurgel et al. 2010). A similar functional overlap of GlnB and GlnK was reported in enteric bacteria (Atkinson and Ninfa 1998; Forchhammer et al. 1999; Jiang et al. 1998; van Heeswijk et al. 2000). On the other hand, GlnB but not GlnK is required to downregulate glutamine synthetase II (GSII) production in $S$. meliloti under nitrogen excess conditions. This implies that, similar to its role in Rhizobium leguminosarum (Amar et al. 1994), GlnB in Rm1021 is essential for dephosphorylating NtrC and the absence of GlnB results in high GSII production. Additionally, mutations in the GlnD protein impaired the ability of $S$. meliloti and $R$. leguminosarum to increase the level of GSII in response to low nitrogen conditions, suggesting that GlnD was required for NtrC phosphorylation, probably through GlnB uridylylation (Schlüter et al. 2000; Yurgel et al. 2010).

The $g \ln D$ gene cannot be deleted in $S$. meliloti (Rudnick et al. 2001; Yurgel and Kahn 2008), implying that GlnD is an essential protein. However, Rm1021 producing an N-terminally truncated GlnD protein, missing 70 amino acids and generated either by the transposon insertion $g \ln D:: \operatorname{Tn} 5-\mathrm{TcW} 1$ or the constructed in-frame deletion mutation $\Delta g \ln D$-sm2, is viable. Both strains have severe defects in NSR, including loss of the ability to utilize many nitrogen sources (Yurgel and Kahn 2008). The only exception is glutamine, which supported moderate growth of Rm1021 $\Delta g \ln D$-sm2 when used as a nitrogen source. Both S. meliloti Rm1021 glnD::Tn5-TcW1 and $\Delta g \ln D$-sm2 mutants fixed substantial nitrogen in symbiosis with alfalfa but the symbiosis was ineffective because the plants could not use the fixed nitrogen for growth, generating a novel Fix ${ }^{+} \mathrm{Eff}^{-}$phenotype (Yurgel and Kahn 2008). On the other hand, a Rm1021 $\Delta g \ln B \Delta g \ln K$ double mutant missing both PII proteins forms an effective symbiosis, indicating that the PII proteins are not required for adequate nitrogen exchange in symbiosis (Yurgel et al. 2010). As expected from its similarity to other GlnD proteins, the amino terminally deleted GlnD in Rm1021 $g \ln D$-sm2 cannot modify PII proteins in vitro, suggesting either that unmodified PII proteins interfere with regulation of nitrogen metabolism in symbiosis or that GlnD is involved in a symbiotically essential process that does not require the PII proteins. 
In the research described here, we tested the ability of a strain containing an unmodified GlnB to form effective nodules and performed a more comprehensive analysis of the role of PII proteins in regulating nitrogen metabolism in S. meliloti. We also dissected the interaction of GlnD and the PII proteins in symbiosis and free-living growth by constructing and characterizing an Rm1021triple $g \ln B / K / D$ mutant.

\section{RESULTS AND DISCUSSION}

There are differences between the phenotypes of the $g \ln D$ sm2 mutant and the $g \ln B g \ln K$ double-deletion strain that were difficult to explain using a model in which the NSR measurement made by GlnD is then transduced by modifying GlnB and GlnK to induce the appropriate cellular responses. One possibility for this difference was that the GlnD-sm2 protein is unable to uridylylate the PII proteins, and strains containing the unmodified PII proteins are different in symbiosis from strains that completely lack the PII proteins. To understand the effect of GlnB uridylylation on nitrogen metabolism in free-living cells of $S$. meliloti, we cloned a $g \ln B$ gene with its 500 -bp promoter region in a low-copy broad host range vector pCPP30 (Huang et al. 1992), producing plasmid pCPPGlnB. In addition, we constructed a plasmid, pCPPGlnBP5, carrying the $g \ln B P 5$ mutation that replaces Tyr51, which is the normal site of uridylylation, with Phe51, resulting in a protein that cannot be modified (Arcondéguy et al. 1997). Structure-function analysis of the Escherichia coli GlnB protein identified a number of mutations that significantly affected its rate of uridylylation and other properties (Arcondéguy et al. 2001). Our choice of the Tyr51Phe mutation was based on the complete inability of the protein to be modified and a previous investigation of the effect of this mutation in S. meliloti (Arcondéguy et al. 1997).

Plasmids pCPPGInB and pCPPGlnBP5 were introduced into the Rm1021 wild-type strain, a strain missing both PII proteins $(\mathrm{Rm} 1021 \Delta g \ln B \Delta g \ln K)$, or a $\Delta g \ln D \mathrm{~N}$-terminal deletion mutant $(\mathrm{Rm} 1021 \Delta g \ln D$-sm2) and the resulting strains were analyzed using an in vitro uridylylation assay that measures the attachment of $\left[\alpha{ }^{32} \mathrm{P}\right]$ UTP to proteins in permeabilized cells (Yurgel et al. 2010). A single approximately $11-\mathrm{kDa}$ band, corresponding to a protein with the size of $\mathrm{GlnB}$ or $\mathrm{GlnK}$, was labeled in Rm1021 incubated with $\alpha$-ketoglutarate (Fig. 2, lane 1) but nothing was labeled in the samples incubated with glutamine (Fig. 2, lane 2). No labeled band was seen in Rm1021 $\Delta g \ln B$ $\Delta g \ln K$ incubated in either $\alpha$-ketoglutarate or glutamine (Fig. 2, lanes 3 and 4). In Rm1021 $\Delta g \ln B \Delta g \ln K(\mathrm{pCPPG} \operatorname{lnB})$, which contains the plasmid carrying the wild-type $G \ln B$ protein, a labeled band was detected in cells incubated with $\alpha$-ketoglutarate but not in the cells incubated with glutamine (Fig. 2, lanes 5 and 6). In contrast, the isogenic strain Rm1021 $\Delta g \ln B \Delta g \ln K$ (pCPPGlnBP5), carrying a GlnB Tyr51Phe mutation that lacks the uridylylation site, did not have a UMP-labeled band (Fig. 2, lanes 7 and 8). These results are consistent with the labeled approximately $11-\mathrm{kDa}$ protein being a PII protein, confirm expression of $\mathrm{G} \ln B$ from the plasmid, and indicate that the GlnBP5 protein cannot be modified.

\section{Growth of strains containing GInB or GInBP5.}

The presence of plasmids carrying wild-type $g \ln B$ or its glnBP5 allele did not affect the growth of Rm1021; strains

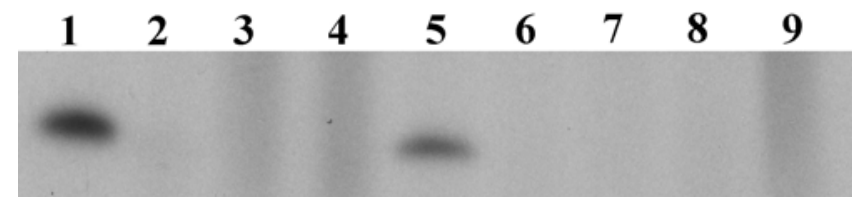

Fig. 2. PII uridylylation in mutants with defects in the nitrogen stress response regulatory cascade. Strains were grown in minimal medium containing glutamate, permeabilized using cetyltrimethylammonium bromide, and incubated with $\left[\alpha-{ }^{32} \mathrm{P}\right]-\mathrm{UTP}$ and $\alpha$-ketoglutarate (lanes $1,3,5$, and 7) or glutamine (lanes 2, 4, 6, and 8). Lanes 1 and 2, Rm1021; lanes 3 and 4, $\mathrm{Rm} 1021 \Delta g \ln B \Delta g \ln K$; lanes 5 and $6, \mathrm{Rm} 1021 \Delta g \ln B \Delta g \ln K(\mathrm{pCPPGlnB})$; lanes 7 and $8, R m 1021 \Delta g \ln B \Delta g \ln K($ pCPPGInBP5).

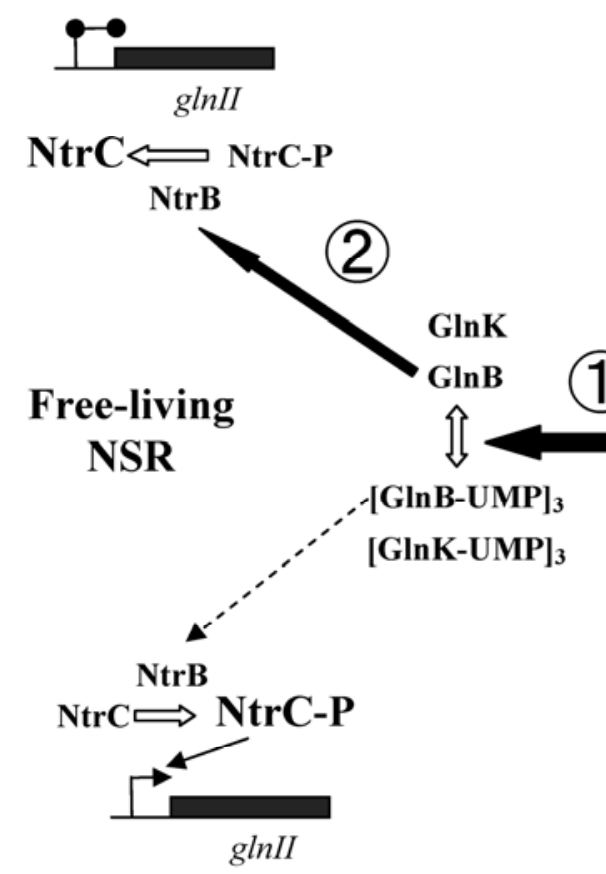

\section{High N}

Glutamine

(3) Free-living GInD and symbiotic function

\section{Ketoglutarate \\ Low N}

Fig 1. Abbreviated version of the nitrogen stress response (NSR) circuitry present in Sinorhizobium meliloti. In response to cellular nitrogen status, the GlnD sensor protein modifies the PII proteins GlnB and GlnK. These transcriptionally and post-translationally regulate the production and activity of many nitrogen-related proteins, including glnII (glutamine synthetase [GS]II). Number 1, GlnD regulates the fraction of the PII proteins (GlnB and GlnK) that are modified with UMP; number 2, unmodified GlnB triggers dephosphorylation of NtrC and represses GSII synthesis; number 3, a conclusion of this article is that GlnD influences free-living and symbiotic properties in a way that does not depend on the PII proteins. 
Rm1021(pCPPGlnB) and Rm1021(pCPPGlnBP5) formed colonies with sizes similar to those of Rm1021 on all nitrogen sources tested (Table 1). However, introducing pCPPGlnB into $\mathrm{Rm} 1021 \Delta g \ln B \Delta g \ln K$ restored a normal growth rate to the $\mathrm{Rm} 1021 \Delta g \ln B \Delta g \ln K$ mutant, which grows extremely slowly. Introducing pCPPGlnBP5 into Rm1021 $\Delta g \ln B \Delta g \ln K$ partially restored the growth of the strain but not to the extent that pCPPGlnB did (Table 1). This limited rescue is consistent with the unmodified state of the GlnBP5 protein, because no UMP modification signals nitrogen sufficiency and the cells do not respond by inducing catabolic pathways. On the other hand, introducing pCPPGInB or pCPPGlnBP5 into Rm1021 $\Delta \ln D$ sm2 did not result in faster growth on any tested nitrogen sources. This was the expected result, because the N-terminally deleted GlnD in Rm1021 $\Delta g \ln D$-sm2 is unable to modify PII proteins (Yurgel et al. 2010) and, whereas the addition of pCPPGlnB or pCPPGlnBP5 might change the amount of GlnB in the $\Delta g \ln D$-sm2 mutant, it would not change the uridylylation state of the PII proteins. The ability of pCPPGInBP5 to improve the growth of $\mathrm{Rm} 1021 \Delta g \ln B \Delta g \ln K$ indicates that unmodified GlnB may play some role in regulating catabolism of a number of nitrogen sources but it does not rescue growth as well as the modifiable form of GlnB. However, the difference in growth on many of the nitrogen sources tested between the strains with a $\Delta g \ln D$-sm2 mutation and $\mathrm{Rm} 1021 \Delta g \ln B \Delta g \ln K$ (pCPPGInBP5) indicates that the defects in the NSR of Rm1021 $\Delta g \ln D$-sm2 cannot simply be explained as a result of locking PII proteins into a deuridylylated state. This implies that the $S$. meliloti GlnD protein influences the NSR in some way that does not depend on the PII proteins.

\section{GSII accumulation in GInBP5 mutants.}

$S$. meliloti and $R$. leguminosarum glnD mutants do not activate transcription of NtrC-dependent genes, including the $g \ln I I$ gene encoding GSII, probably because of permanently deuridylylated PII proteins (Amar et al. 1994; Yurgel et al. 2010). To test this hypothesis, we measured the effect of GlnBP5, the unmodifiable form of GlnB, on GSII accumulation in $\mathrm{Rm} 1021 \Delta g \ln B \Delta g \ln K$, which constitutively overproduces GSII (Yurgel et al. 2010). When GSII antibodies were used to stain Western blots of protein extracts from Rm1021 cells grown on glutamate, a band was visible at approximately $36 \mathrm{kDa}$, the size of GSII monomer. The band was not seen in cells grown on ammonium. The nitrogen source does not make a difference to Rm1021 $\Delta g \ln B \Delta g \ln K$, which has the band in both glutamate- and ammonium-grown cells (Fig. 3A). We have evidence that this may be due to activation of NtrC phosphorylation by another two-component regulatory system (K. Hagberg, M. Mulder, M. L. Kahn, and S. N. Yurgel, unpublished data).

Introduction of pCPPGlnB into Rm1021 $\Delta g \ln B \Delta g \ln K$ restored the normal regulation of GSII expression-crude extracts of $\mathrm{Rm} 1021 \Delta g \ln B \Delta g \ln K(\mathrm{pCPPG} \operatorname{lnB})$ produced GSII under low-nitrogen (glutamate) but not under high-nitrogen (ammonium) conditions (Fig. 3A). In contrast, the band corresponding to the GSII protein was detected when Rm1021 $\Delta g \ln B$ $\Delta g \ln K(\mathrm{pCPPG} \ln \mathrm{BP} 5)$ cells were grown on either glutamate or ammonium (Fig. 3A). We had expected that unmodified GlnB would repress GSII production in Rm1021 under both conditions because the lack of modification is characteristic of high nitrogen availability (Yurgel and Kahn 2008; Yurgel et. al. 2010). The presence of pCPPGlnB or pCPPGlnBP5 had no effect on the lack of GSII accumulation in Rm1021 $\Delta g \ln D$-sm2 (Fig. 3A). Although this was consistent with the prediction of the standard model that the GlnD-sm2 protein does not modify the PII proteins and would lead to lower levels of GSII, the difference between this lack of GSII and the accumulation of GSII seen in Rm1021 $\Delta g \ln B \Delta g \ln K(\mathrm{pCPPG} \operatorname{lnBP5})$ was striking.

\section{Symbiotic phenotype of GInBP5 mutants.}

The difference between the symbiotic phenotypes of $\mathrm{Rm} 1021 \Delta g \ln D$-sm 2 and $\mathrm{Rm} 1021 \Delta g \ln B \Delta g \ln K$ could indicate that the presence of the nonuridylylated PII proteins in $\mathrm{Rm} 1021 \ln D$-sm2 interfered with the regulation of nitrogen exchange in symbiosis (Yurgel and Kahn 2008; Yurgel et al.

Table 1. Growth properties of Sinorhizobium meliloti strains ${ }^{\mathrm{a}}$

\begin{tabular}{|c|c|c|c|c|c|c|c|c|c|c|}
\hline $\begin{array}{l}\text { Nitrogen } \\
\text { source }^{b}\end{array}$ & $\operatorname{Rm} 1021$ & $\begin{array}{c}\mathrm{Rm} 1021 \\
\Delta g \ln D-\mathrm{sm} 2\end{array}$ & $\begin{array}{c}\operatorname{Rm} 1021 \\
\Delta g \ln D-\operatorname{sm} 2 \\
(\mathrm{pCPPG} \ln \mathrm{B})\end{array}$ & $\begin{array}{c}\text { Rm1021 } \Delta g l n D- \\
\text { sm2 } \\
(\text { pCPPGlnBP5) }\end{array}$ & $\begin{array}{c}\mathrm{Rm} 1021 \\
\Delta g \ln B \Delta g \ln K\end{array}$ & $\begin{array}{c}\mathrm{Rm} 1021 \\
\Delta g \ln B \Delta g \ln K \\
(\mathrm{pCPPG} \ln \mathrm{B})\end{array}$ & $\begin{array}{c}\mathrm{Rm} 1021 \\
\Delta g \ln B \Delta \sin K \\
(\mathrm{pCPPG} \ln B P 5)\end{array}$ & $\begin{array}{c}\mathrm{Rm} 1021 \Delta g l \\
n B \Delta g \ln K \\
g \ln D:: \operatorname{Tn} 5- \\
\mathrm{TcW} 1\end{array}$ & $\begin{array}{c}\mathrm{Rm} 1021 \Delta g \ln B \Delta \\
g \ln K \operatorname{gln} D:: \operatorname{Tn} 5- \\
\mathrm{TcW} 1 \\
(\mathrm{pCPPG} B)\end{array}$ & $\begin{array}{c}\mathrm{Rm} 1021 \Delta g \ln B \Delta \\
g \ln K \operatorname{gln} D:: T n 5- \\
\text { TcW1 } \\
(\mathrm{pCPPGInBP5)}\end{array}$ \\
\hline Adenosine & 5 & 2 & 2 & 2 & 2 & 5 & 3 & 0 & 2 & 2 \\
\hline Alanine & 5 & 2 & 2 & 2 & 2 & 5 & 3 & 0 & 2 & 2 \\
\hline Arginine & 5 & 2 & 2 & 2 & 2 & 5 & 3 & 0 & 2 & 2 \\
\hline Asparagine & 5 & 2 & 2 & 2 & 2 & 5 & 3 & 0 & 2 & 2 \\
\hline Aspartate & 5 & 2 & 2 & 2 & 2 & 5 & 3 & 0 & 2 & 2 \\
\hline Cysteine & 4 & 0 & 0 & 0 & 2 & 4 & 2 & 0 & 0 & 0 \\
\hline GABA & 5 & 0 & 0 & 0 & 2 & 5 & 2 & 0 & 0 & 0 \\
\hline Glucosamine & 5 & 0 & 0 & 0 & 1 & 5 & 2 & 0 & 0 & 0 \\
\hline Glutamate & 5 & 2 & 2 & 2 & 2 & 5 & 4 & 0 & 2 & 2 \\
\hline Glutamine & 5 & 4 & 4 & 4 & 2 & 5 & 4 & 0 & 4 & 4 \\
\hline Glycine & 5 & 0 & 0 & 0 & 2 & 5 & 3 & 0 & 0 & 0 \\
\hline Histidine & 5 & 2 & 2 & 2 & 2 & 5 & 3 & 0 & 2 & 2 \\
\hline Isoleucine & 4 & 2 & 2 & 2 & 1 & 2 & 1 & 0 & 2 & 2 \\
\hline Leucine & 5 & 2 & 2 & 2 & 2 & 4 & 3 & 0 & 2 & 2 \\
\hline Lysine & 5 & 2 & 2 & 2 & 2 & 5 & 2 & 0 & 2 & 2 \\
\hline Methionine & 4 & 1 & 1 & 1 & 1 & 5 & 3 & 0 & 1 & 1 \\
\hline${\mathrm{MM}-\mathrm{NH}_{4}}_{4}$ & 5 & 2 & 2 & 2 & 3 & 5 & 3 & 0 & 2 & 2 \\
\hline Phenylalanine & 4 & 0 & 0 & 0 & 0 & 1 & 0 & 0 & 0 & 0 \\
\hline Proline & 5 & 1 & 1 & 1 & 2 & 5 & 4 & 0 & 1 & 1 \\
\hline Serine & 5 & 0 & 1 & 0 & 2 & 5 & 3 & 0 & 0 & 0 \\
\hline Taurine & 4 & 0 & 0 & 0 & 1 & 1 & 1 & 0 & 0 & 0 \\
\hline Tryptophan & 4 & 0 & 0 & 0 & 0 & 1 & 0 & 0 & 0 & 0 \\
\hline Tyrosine & 4 & 0 & 0 & 0 & 0 & 1 & 0 & 0 & 0 & 0 \\
\hline Valine & 5 & 1 & 1 & 1 & 1 & 5 & 3 & 0 & 1 & 1 \\
\hline YMB & 5 & 4 & 4 & 4 & 5 & 5 & 5 & 2 & 4 & 4 \\
\hline
\end{tabular}

\footnotetext{
${ }^{a}$ Strains were grown on solid agar plates for 7 days. Growth rate shown from high $=5$ to low $=0$.
}

${ }^{\mathrm{b}} \mathrm{GABA}=\gamma$-amino-n-butyric acid, $\mathrm{MM}=$ minimal-mannitol, and $\mathrm{YMB}=$ yeast mannitol broth. 
2010). To test this possibility, we analyzed the symbiotic phenotype of $\mathrm{Rm} 1021 \Delta g \ln B \Delta g \ln K(\mathrm{pCPPG} \operatorname{lnBP} 5)$ and found that it established an effective symbiosis with alfalfa; the strain formed pink, healthy nodules and the plants were green and similar in mass to plants inoculated with Rm1021 $\Delta g \ln B \Delta g \ln K$ or $\mathrm{Rm} 1021 \Delta g \ln B \Delta g \ln K(\mathrm{pCPPG} \operatorname{lnB})$ (Table 2). Introducing pCPPGlnB or pCPPGlnBP5 also did not alter the symbiotic phenotypes of Rm1021 or Rm1021 $\operatorname{Rg} \ln D$-sm2 (Table 2). Our earlier results showed that PII proteins do not need to be present for a normal symbiosis; these results show that unmodified GlnB, characteristic of sufficient nitrogen in free-living bacteria, does not interfere with nitrogen exchange in symbiosis.

\section{Construction of a triple $\Delta g \ln B \Delta g \ln K g \ln D$-sm2 mutant.}

To further dissect the interaction of the different components of the NSR cascade in free-living cells and in the symbiotic association of Rm1021 with alfalfa, we attempted to introduce an in-frame $g \ln D$-sm2 deletion into the Rm1021 $\Delta g \ln B \Delta g \ln K$ mutant using the same recombination-replacement strategy that we used to construct the Rm1021 $\ln \ln$-sm2 strain in Rm1021 (Yurgel and Kahn 2008). Several attempts were unsuccessful (Yurgel et al. 2010). However, we have now succeeded in transducing the $g \ln D:: \operatorname{Tn} 5-\mathrm{TcW} 1$ transposon mutation from the TcW1 strain into Rm1021 $\operatorname{Rg} \ln B \Delta g \ln K$. The $g \ln D:: \mathrm{Tn} 5-$ TcW1 mutation also removes the N-terminal part of GlnD. TcW1 and Rm1021glnD-sm2 have similar free-living and symbiotic phenotypes (Yurgel and Kahn 2008). Both strains fix atmospheric dinitrogen but the host plant cannot use this nitrogen for growth. TcW1 also has growth properties similar to Rm1021 $\ln D$-sm2-it grows poorly on all nitrogen sources tested, with the exception of glutamine.

\section{Free-living phenotypes of the triple $g \ln B K D$ mutant.}

The resulting triple mutant, $\mathrm{Rm} 1021 \Delta g \ln B \Delta g \ln K g \ln D:: \mathrm{Tn} 5$ TcW1, grew extremely poorly on Luria-Bertani (LB) media and could not grow on any other tested media (Table 1). This phenotype was more severe than that of the double $\Delta g \ln B \Delta g \ln K$ or single $g \ln D:: \mathrm{Tn} 5-\mathrm{TcW} 1$ (or $g \ln D$-sm2) mutants seen earlier. This growth defect probably explains our inability to isolate the Rm1021 $\Delta g \ln B \Delta g \ln K \Delta g \ln D$-sm2 mutant,
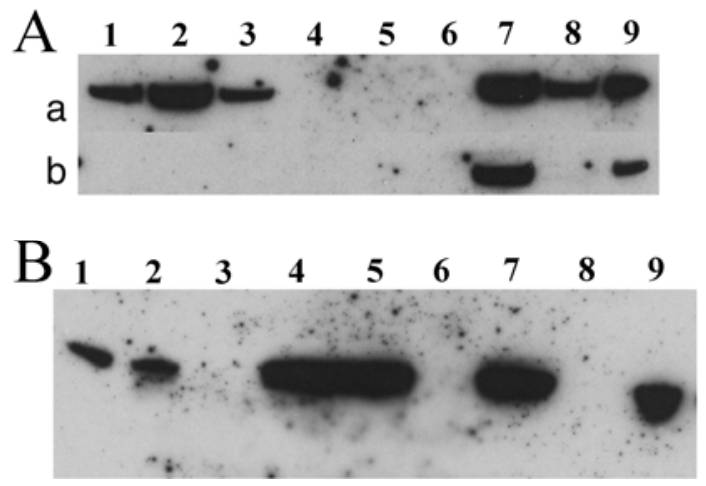

Fig. 3. A, Production of glutamine synthetase [GS]II in mutants with defects in the nitrogen stress response regulatory cascade. GSII was visualized in cells grown in media that contained row A, glutamate or row B, ammonium as nitrogen source. Lane 1, Rm1021; lane 2, Rm1021 (pCPPGlnB); lane 3, Rm1021(pCPPGlnBP5); lane 4, Rm1021 $\ln \ln D$ sm2; lane 5, Rm1021 $\Delta g \ln D$-sm2(pCPPGlnB); lane 6, Rm1021 $\Delta g \ln D$-sm2 (pCPPGlnBP5); lane 7, Rm1021 $\Delta g \ln B \Delta g \ln K$; lane 8, Rm1021 $\Delta \ln B$ $\Delta g \ln K(\mathrm{pCPPG} \operatorname{lnB})$; lane 9, Rm1021 $\ln \ln B \Delta g \ln K(\mathrm{pCPPGlnBP5}) . \mathbf{B}$, GSII was visualized in cells grown in media that contained glutamate (lanes 1 to 5) or ammonium (lanes 6-9) as nitrogen source. Lanes 1, 6, Rm1021; lanes 2 and 7, Rm1021 $\Delta g \ln B \Delta g \ln K$; lanes 3 and 8, Rm1021 $\Delta g \ln D$-sm2; lanes 4 and 9, Rm1021 $\Delta g \ln B \Delta g \ln K g \ln D:: \mathrm{Tn} 5-\mathrm{TcW} 1$, clone number 1; lane 5, Rm1021 $\ln \ln B \Delta g \ln K g \ln D:: \operatorname{Tn} 5-\mathrm{TcW} 1$, clone number 2. because the final step in the isolation procedure we used requires cells to grow on yeast mannitol broth (YMB) media supplemented with $5 \%$ sucrose.

Because the triple mutant was unable to grow on minimal media with either glutamate or ammonium as nitrogen source, we modified our normal procedure to analyze GSII production by the strain. We first grew Rm1021 $\Delta g \ln B \Delta g \ln K g \ln D:: T n 5-$ TcW1 in LB broth for $24 \mathrm{~h}$, harvested the cells by centrifugation, resuspended them in minimal-mannitol (MM)- $\mathrm{NH}_{4}{ }^{+}$or MM-glutamate media, and incubated the cultures overnight. Western blot analysis of GSII accumulation showed that $\mathrm{Rm} 1021 \Delta g \ln B \Delta g \ln K g \ln D:: \mathrm{Tn} 5-\mathrm{TcW} 1$ produced a large amount of GSII protein under both low-nitrogen (glutamate) and highnitrogen (ammonium) conditions (Fig. 3B, lanes 4, 5, and 9). The strain resembles $\mathrm{Rm} 1021 \Delta g \ln B \Delta g \ln K$, indicating that that the absence of the PII proteins plays a dominant role with respect to regulation of GSII production. For GSII production, the $\Delta g \ln B \Delta g \ln K$ mutations are dominant to the $g \ln D:: \operatorname{Tn} 5-$ TcW1 mutation.

Introducing pCPPGInB or pCPPGInBP5 into Rm1021 $\Delta g \ln B$ $\Delta g \ln K g \ln D:: \operatorname{Tn} 5-\mathrm{TcW} 1$ slightly improved the growth of the resulting strains but both plasmid-containing strains grew similarly. This is consistent with the idea that the GlnD defect blocks GlnB modification so that the actual form of GlnB in both strains is similar.

\section{Symbiotic phenotype of the triple mutant.}

The symbiotic performance of the triple mutant was analyzed using alfalfa plants. The mutant formed pink, healthylooking nodules on the host plant roots (Fig. 4). Analysis of ${ }^{15} \mathrm{~N}_{2}$ accumulation showed that nodules were fixing atmospheric $\mathrm{N}_{2}$ at the same rate as the nodules formed by the parental Rm1021 strain (Table 3). However, the plants nodulated by Rm1021 $\Delta g \ln B \Delta g \ln K g \ln D:: T n 5-\mathrm{TcW} 1$ did not benefit from this association. The plants were small and yellow, with mass similar to the uninoculated control (Table 2; Fig. 4). The plants had red stems, which are characteristic of anthocyanin production in stressed plants. Similar phenotypes were described for the single Rm1021 glnD mutants, Rm1021 $\operatorname{Rg} \ln D-s m 2$ and TcW1 (Yurgel and Kahn 2008). In addition, the expression of GlnB or GlnBP5 proteins had no effect on the symbiotic phenotype of $\mathrm{Rm} 1021 \Delta g \ln B \Delta g \ln K g \ln D:: \mathrm{Tn} 5-\mathrm{TcW} 1$ (Table 2). These results clearly demonstrated that, although PII proteins play an important role in regulating the NSR in S. meliloti Rm1021 free-living cells, their function was not critical in establishing a nitrogen-fixing symbiotic association that supports the growth of the host plant. In symbiosis, the $g \ln D:: \operatorname{Tn} 5$ TcW1 mutation was dominant to the $\Delta g \ln B \Delta g \ln K$ mutations.

Table 2. Symbiotic phenotype of Sinorhizobium meliloti strains

\begin{tabular}{|c|c|}
\hline Strains & $\begin{array}{c}\text { Plant shoot dry } \\
\text { mass (mg/plant) }\end{array}$ \\
\hline Rm1021 & $10.8 \pm 2.19$ \\
\hline No inoculation & $4.1 \pm 0.82$ \\
\hline Rm1021(pCPPGlnB) & $12.7 \pm 1.69$ \\
\hline Rm1021(pCPPGlnBP5) & $11.2 \pm 2.42$ \\
\hline $\mathrm{Rm} 1021 \Delta g \ln B \Delta g \ln K$ & $10.9 \pm 1.20$ \\
\hline $\mathrm{Rm} 1021 \Delta g \ln B \Delta g \ln K(\mathrm{pCPPG} \ln \mathrm{B})$ & $12.5 \pm 2.84$ \\
\hline $\mathrm{Rm} 1021 \Delta g \ln B \Delta g \ln K(\mathrm{pCPPG} \operatorname{lnBP5})$ & $10.1 \pm 1.82$ \\
\hline $\mathrm{Rm} 1021 \Delta g \ln D-\mathrm{sm} 2$ & $6.9 \pm 0.65$ \\
\hline $\mathrm{Rm} 1021 \Delta g \ln D-\mathrm{sm} 2(\mathrm{pCPPG} \operatorname{lnB})$ & $4.8 \pm 0.56$ \\
\hline $\mathrm{Rm} 1021 \Delta g \ln D$-sm2(pCPPGlnBP5) & $5.1 \pm 1.16$ \\
\hline $\mathrm{Rm} 1021 \Delta g \ln B \Delta g \ln K g \ln D:: \mathrm{Tn} 5-\mathrm{TcW} 1$ & $5.4 \pm 0.41$ \\
\hline $\mathrm{Rm} 1021 \Delta g \ln B \Delta g \ln K g \ln D:: \mathrm{Tn} 5-\mathrm{TcW} 1(\mathrm{pCPPG} \operatorname{lnB})$ & $6.6 \pm 1.31$ \\
\hline \multirow{2}{*}{\multicolumn{2}{|c|}{$\begin{array}{l}\mathrm{Rm} 1021 \Delta g \ln B \Delta g \ln K g \ln D:: \mathrm{Tn} 5-\mathrm{TcW} 1 \\
\quad(\mathrm{pCPPG} \ln B \mathrm{P} 5)\end{array}$}} \\
\hline & $6.8 \pm 1.40$ \\
\hline
\end{tabular}

${ }^{\text {a }}$ Values are the mean and standard deviation for four replicate measurements of six plants from the Magenta box (BioWorld, Dublin. OH, U.S.A. 


\section{Conclusion.}

In the standard model of NSR regulation, GlnD plays an important role in regulating bacterial response to nitrogen availability as the sensor that initiates a modification cascade that controls nitrogen assimilation and catabolism activities at both the transcriptional and post-translational levels (Fig. 1). Recently, we showed that $g \ln D$-sm2, a mutation that is expected to generate an N-terminally truncated GlnD mutant protein, was defective in inducing an NSR and, whereas the mutation did not interfere with symbiotic nitrogen fixation, it blocked normal nitrogen exchange in the symbiosis between $S$. meliloti Rm1021 and alfalfa (Yurgel and Kahn 2008).

The aim of this study was to dissect the function of GlnD and $\mathrm{GlnB} / \mathrm{GlnK}$ proteins in free-living and symbiotic forms of $\mathrm{Rm} 1021$. The results presented above are consistent with the idea that GlnD can communicate with the cell in some way that does not depend on the PII proteins. Previous experiments with Rm1021 $g \ln B$ and $g \ln K$ mutants showed that the loss of either or both of these genes did not lead to the same phenotype as the $g \ln D$-sm2 mutation (Yurgel et al. 2010). One possibility that we had not eliminated was that the unmodified form of the PII proteins that would be found in the $g \ln D$-sm 2 mutant strain might be responsible for the symbiotic phenotype. We investigated this by introducing a plasmid carrying the $g \ln B$ Tyr51Phe mutation into a $\Delta g \ln B \Delta g \ln K$ strain. Although the plasmid does affect some free-living growth phenotypes, it does not block the effectiveness of the strain in symbiosis, as would have been expected if unmodified GlnB was important.

We considered it possible that more than one mechanism might be responsible for the Fix $^{+} \mathrm{Eff}^{-}$symbiotic phenotype.
The GlnB Tyr51Phe experiments test the idea that having only unmodified PII proteins will lead to a Fix ${ }^{+} \mathrm{Eff}^{-}$symbiotic phenotype, while the triple-mutant experiments examined the idea that a GlnD unable to carry out the uridylylation reaction would be suppressed epistatically by the absence of any substrates. In $E$. coli, a $g \ln B K D$ triple mutant has growth properties similar to the isogenic $g \ln B K$ strain (Atkinson and Ninfa 1998). These results were interpreted to mean that all of the $g \ln D$ phenotypes examined were due to GlnD modifying the GlnB/GlnK proteins and that, when these proteins were missing in the $g \ln B K$ mutant, a $g \ln D$ mutation had no effect. The observation that $\mathrm{Rm} 1021 \Delta g \ln B \Delta g \ln K g \ln D:: \mathrm{Tn} 5-\mathrm{TcW} 1$ has a more severe growth defect than either $\mathrm{Rm} 1021 \Delta g \ln B \Delta g \ln K$ or $\mathrm{Rm} 1021 \Delta g \ln D$-sm2 supports our conclusion that GlnD plays other roles in free-living $S$. meliloti cells besides controlling PII protein modification. In the $g \ln B g \ln K g \ln D:: T n 5-\mathrm{TcW} 1$ mutant, the inability of the $g \ln B g \ln K$ mutations to block the Fix $^{+} \mathrm{Eff}^{-}$phenotype of the $g \ln D:: \mathrm{Tn} 5-\mathrm{TcW} 1$ mutation was further evidence of an interaction not predicted by the classical NSR. The epistatic relationships were interesting-Rm1021$\Delta g \ln B \Delta g \ln K \Delta g \ln D$-sm2 resembled $\mathrm{Rm} 1021 \Delta g \ln B \Delta g \ln K$ in pro-

Table 3. ${ }^{15} \mathrm{~N}$ incorporation in shoots, roots, and nodules ${ }^{\mathrm{a}}$

\begin{tabular}{|c|c|c|}
\hline & Rm1021 & Rm1021 $\Delta g \ln B \Delta g \ln K g \ln D:: T n 5-T c W 1$ \\
\hline Nodules & $497 \pm 44.7$ & $843 \pm 395.7$ \\
\hline Roots & $268 \pm 1.9$ & $68.6 \pm 1.70$ \\
\hline Shoot & $26.3 \pm 3.32$ & $11.5 \pm 0.21$ \\
\hline
\end{tabular}

a Values $\left(\partial^{15} \mathrm{~N} / \mathrm{mg}\right.$ tissue) are the mean and standard deviation for three replicate measurements of samples pooled from 18 plants.

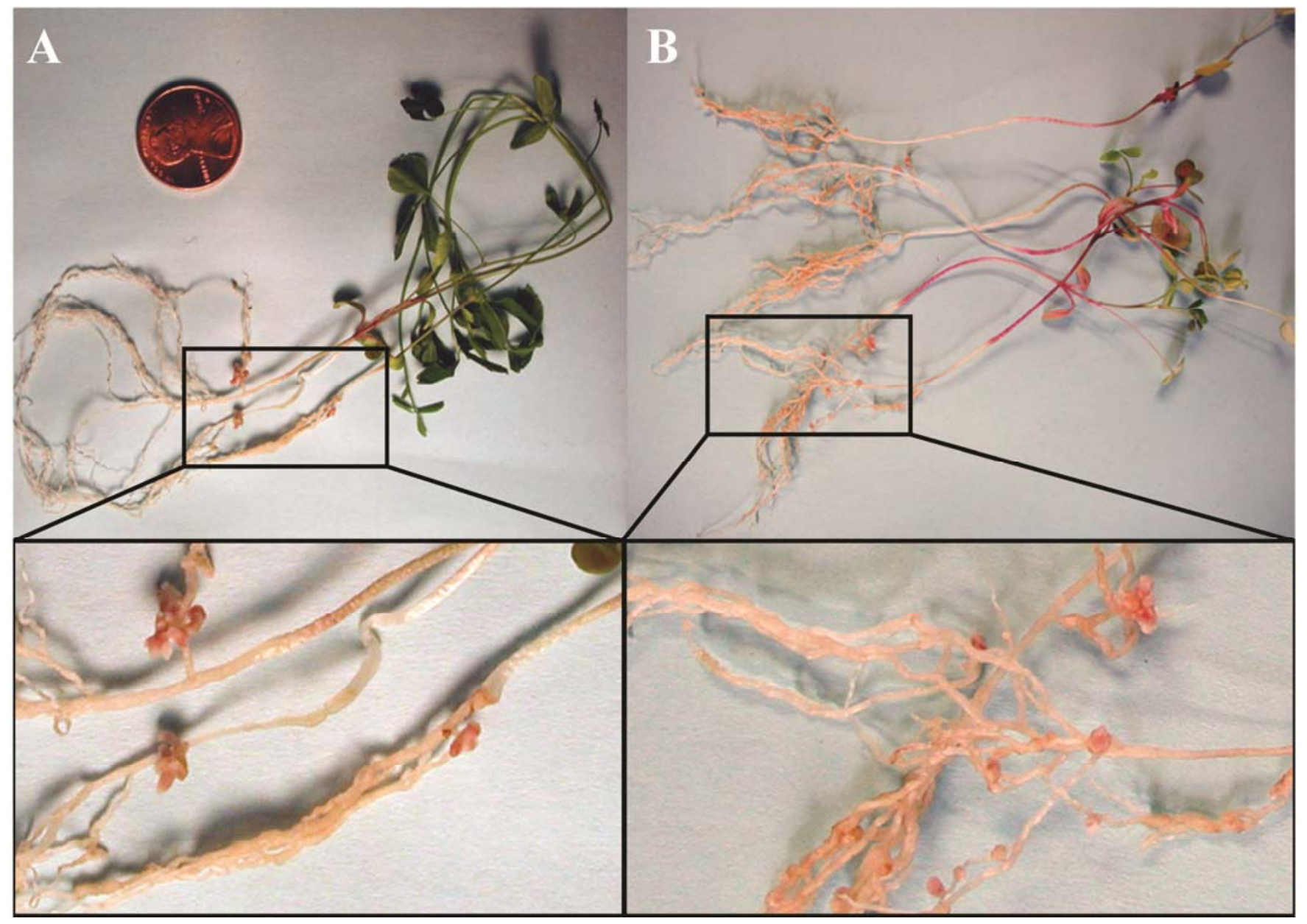

Fig. 4. Alfalfa plants nodulated with Sinorhizobium meliloti strains A, Rm1021 and B, Rm1021 $\operatorname{Rin} B \Delta g \ln K g \ln D:: \mathrm{Tn} 5-\mathrm{TcW} 1$. 
ducing GSII in free-living growth but the symbiotic phenotype of the triple mutant was clearly that of the $\Delta g \ln D$-sm2 mutant.

The components of the standard NSR are all present in $S$. meliloti but, in addition to regulating NSR, our data suggest that GlnD interacts with something besides the PII proteins. There are few reports of $g \ln D$ mutant phenotypes that are not related to the NSR. Graf and Ruby (2000) showed that a transposon mutation in the Vibrio fischerii $g \ln D$ affected iron utilization and symbiotic competence in its interaction with the Hawaiian squid. Tøndervik and associates (2006) isolated transposon mutations in $E$. coli glnD that suppress the osmotic sensitivity caused by other mutations, suggesting a role in osmotic regulation. These mutations do not block the uridyltransferase activity. And, although Sun and associates (2002) found that mutations in Azospirillum brasiliensis $g \ln D, g \ln B$, and $g \ln Z$ (a third PII protein) alter polyhydroxybutyrate (PHB) synthesis, they interpreted this as a secondary consequence of disrupting normal NSR control.

The role of GlnB and GlnK PII proteins in NSR regulation is well documented (Arcondéguy et al. 1996, 1997, 2001; Atkinson et al. 1994, 2002; Ninfa and Atkinson 1998, 2000; Reitzer et al. 2003; Yurgel et al. 2010). A close examination of the genome sequence did not suggest the presence of any other PII proteins in this $S$. meliloti strain. In addition, no other proteins appear to be modified in the permeabilized cell assay used to measure GlnD uridylyltransferase. The two PII proteins in $S$. meliloti were studied by construction of i) two $g \ln B$ mutants in strain GMI708 carrying either $\Delta g \ln B 10$, a nonpolar deletion mutation, or $g \ln B P 5$, an allele encoding the Tyr51Phe mutation that removes the tyrosine used in GlnB uridylylation (Arcondéguy et al. 1997); and ii) double nonpolar $g \ln B$ and glnK deletions in Rm1021 (Yurgel and Kahn 2008; Yurgel et al. 2010). The most striking phenotype of these strains is the slower growth of the double mutant; whereas the single mutants have distinct phenotypes, the crosstalk regulation by PII proteins seen in other bacteria is also seen in S. meliloti.

Mutational and biochemical studies indicate that GlnD has several functional domains. Zhang and associates (2010) located four of these: an N-terminal domain nucleotidyltransferase related to DNA polymerases that modifies the PII proteins in response to nitrogen stress, a metal-dependent phosphohydrolase $\mathrm{HD}$ domain in the center of the protein that is involved in removing UMP from modified PII proteins, and two C-terminal sets of repeated ACT sequences like those often found on enzymes that respond to amino acid concentrations. Two other homologies exist in the GlnD sequence: a conserved domain that is characteristic of GlnD proteins and a domain also found in inosine monophosphate dehydrogenases that overlaps the GlnD and phosphohydrolase domains. No distinct function has been assigned to these last two domains, although the second overlaps the HD region that removes nucleotide modifications. One Fix ${ }^{+} \mathrm{Eff}^{+} \mathrm{Tn} 5$ mutation we isolated appears to be near the $\mathrm{C}$ terminus of the HD domain at amino acid 608 (Yurgel and Kahn 2008) but we have not determined whether this mutation affects UMP removal. The mutation causes significant accumulation of glycogen but does not have a strong effect on the symbiosis or NSR phenotypes we examined.

The difference between $S$. meliloti and the enteric bacteria is likely to be in the use that $S$. meliloti makes of GlnD. An $S$. meliloti glnD deletion mutation lacking the entire gene could not be isolated (Rudnick et al. 2001; Yurgel and Kahn 2008), although $g \ln D$ transposon mutants have been isolated in several rhizobia, including $S$. meliloti. The downstream parts of the NSR regulation, represented by $n t r C, g \ln B$, and $g \ln K$, are all dispensable.

An $E$. coli $g \ln D$ deletion mutant has been isolated (Baba et al. 2006). A $g \ln D$ deletion originally could not be isolated in Azotobacter vinelandii and this was traced to excessive adenylylation of the GlnA glutamine synthetase combined with an inability of the mutant to import glutamine (Colnaghi et al. 2001). Rudnick and associates (2001) concluded that GSI adenylylation does not prevent the isolation of a $g \ln D$ deletion in $S$. meliloti by introducing a mutated GSI that could not be adenylylated and showing that this does not allow construction of a complete $g \ln D$ deletion mutation. Although GSI is downregulated in the $\Delta g \ln D$-sm2 mutant, there is some expression and approximately half of the enzyme appears to be unmodified (Yurgel and Kahn 2008).

In this study, we showed that the $\Delta g \ln D$-sm2 mutant had severe defects in regulating free-living and symbiotic nitrogen metabolism compared with Rm1021 $\Delta g \ln B \Delta g \ln K(\mathrm{pCPPG} \operatorname{lnBP5})$, a strain that was designed to mimic the expected state of GlnB in the NSR signal cascade in a $\Delta g \ln D$-sm 2 mutant. These results suggest that the GlnD protein might have other functions in addition to measuring the cell's nitrogen status and communicating this information via the PII-related NSR regulatory cascade (Fig. 1). Moreover, the triple $g \ln B / K / D$ mutant exhibited a Fix ${ }^{+}$fff $^{-}$symbiotic phenotype similar to Rm1021 $\Delta g \ln D$ $s m 2$ while the strain missing both PII proteins still produced an effective Fix $^{+} \mathrm{Eff}^{+}$symbiosis. This suggests either that the GlnD uridylyltransferase is required for proper regulation of nitrogen exchange in symbiosis between Rm1021 and the host plant but that the PII proteins are not involved in this regulation or that the $\Delta g \ln D$-sm2 mutation disrupts some additional activity of GlnD. Analysis of expression patterns in the various mutants isolated here may reveal what aspects of $S$. meliloti are different in the $\Delta g \ln D$-sm 2 mutant and strains that contain an unmodified GlnB protein.

Table 4. Bacterial strains and plasmids

\begin{tabular}{|c|c|c|}
\hline Strains & Genotype or characteristics $^{\mathbf{a}}$ & Source \\
\hline \multicolumn{3}{|l|}{ Sinorhizobium meliloti } \\
\hline Rm1021 & Wild type & Galibert et al. 2001 \\
\hline $\mathrm{Rm} 1021 \Delta g \ln D$-sm2 & $g \ln D$ deletion mutant of $\mathrm{Rm} 1021$ & Yurgel and Kahn 2008 \\
\hline $\mathrm{Rm} 1021 \Delta g \ln K \Delta g \ln B$ & $g \ln K / g \ln B$ deletion mutant of $\mathrm{Rm} 1021$ & Yurgel et al. 2010 \\
\hline $\mathrm{TcW} 1$ & $g \ln D 210 *:: \operatorname{Tn} 5-m o b$ mutant of $\mathrm{Rm} 1021$ & Yurgel and Kahn 2008 \\
\hline $\mathrm{Rm} 1021 \Delta g \ln B \Delta g \ln K g \ln D:: \mathrm{Tn} 5-\mathrm{TcW} 1$ & Transduction of Tn5-mob from TcW1 into Rm1021 $\Delta g \ln B \Delta g \ln K$ & This work \\
\hline GMI708 & $\operatorname{Rif}^{\mathrm{r}}$ derivative of strain $\mathrm{Rm} 2011$ & Batut et al. 1985 \\
\hline GMI3107 & GMI708 glnBP5, Rif, Gm & Arcondéguy et al. 1997 \\
\hline \multicolumn{3}{|l|}{ Escherichia coli } \\
\hline S17-1 & pro $h s d R$ recA [RP4-2(Tc::Mu) (Km::Tn7)] & Simon et al. 1983 \\
\hline \multicolumn{3}{|c|}{$x^{2}$} \\
\hline pCPP30 & IncP1 LacZ; Tc & Huang et al. 1992 \\
\hline pCPPGlnB & $\mathrm{pCPP} 30[\mathrm{Rm} 1021 \mathrm{~g} \ln B]$ & This work \\
\hline pCPPGlnBP5 & pCPP30[GMI3107 $g \ln B]$ & This work \\
\hline
\end{tabular}

${ }^{\mathrm{a}} \mathrm{Rif}^{\mathrm{r}}=$ rifampicin-resistant, $\mathrm{Gm}=$ gentamycin, and $\mathrm{Tc}=$ tetracycline. 


\section{MATERIALS AND METHODS}

\section{Bacterial strains, plasmids, and media.}

The bacterial strains and plasmids used in this study are listed in Table 4. S. meliloti strains were grown at $30^{\circ} \mathrm{C}$ on $\mathrm{LB}$

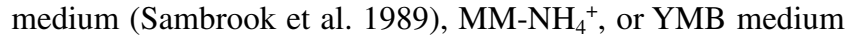
(Somerville and Kahn 1983). E. coli strains were grown at $37^{\circ} \mathrm{C}$ on LB medium. Antibiotics for $S$. meliloti were added at $200 \mu \mathrm{g} / \mathrm{ml}$ (streptomycin), $200 \mu \mathrm{g} / \mathrm{ml}$ (neomycin), or $10 \mu \mathrm{g} / \mathrm{ml}$ (tetracycline). For E. coli, antibiotics were added at $10 \mu \mathrm{g} / \mathrm{ml}$ (tetracycline) or $40 \mu \mathrm{g} / \mathrm{ml}$ (kanamycin).

\section{Transposon transduction.}

Transducing-phage $\Phi \mu 12$ lysates grown on the TcW1 mutant were prepared as described previously (Finan et al. 1984), except that the phage lysate was separated from the lysed cell debris by filtering through a $0.2-\mu \mathrm{m}$ syringe filter (Nalgene, Fisher Scientific, Santa Clara, CA, U.S.A.). Transductants of $\mathrm{Rm} 1021 \Delta g \ln B \Delta g \ln K$ containing the Tn5 mutations in TcW1 were selected on LB medium containing neomycin and purified.

\section{Uridylylation of PII.}

PII uridylylation was examined in permeabilized cells by following the incorporation of radioactivity from $\left[\alpha-{ }^{32} \mathrm{P}\right] \mathrm{UTP}$ into proteins using a modified procedure of Colonna-Romano and associates (1993) as described previously (Yurgel et al. 2010).

\section{Growth measurements.}

To evaluate growth on various carbon substrates, $S$. meliloti cells were suspended in Min-salt (minimal media without carbon and nitrogen source) solution to an optical density at $600 \mathrm{~nm}=0.5$ and the cell suspensions were diluted with Min-salt solution in 1 to 10,1 to 100,1 to $1,000,1$ to 10,000 , and 1 to 100,000 times in a 96-well microplate. Aliquots of these were then transferred using a sterile bolt replicator onto plates containing solid MM to which the indicated nitrogen compound at $0.2 \mathrm{~g} /$ liter had been added. After 3 to 7 days the sizes of isolated colonies were scored. This method of evaluating single isolated colonies is more reliable than measuring bulk growth rates in liquid because pseudorevertants with faster growth can be seen on plates but can easily overgrow the original mutants in liquid culture. The set of potential nitrogen sources included alanine, proline, histidine, glycine, $\gamma$-amino-n-butyric acid, glutamate, glutamine, ammonium, and nitrate.

\section{Construction of $\operatorname{gln} B$ and $g \ln B P 5$ expression plasmids.} $g \ln B$ genes with 500-bp flanking regions were cloned from Rm1021 or GLMI3107 into plasmid pCPP30, a low-copy RK2 derivative that can replicate in $S$. meliloti (Huang et al. 1992). S. meliloti Rm1021 or GMI3107 DNA was amplified by polymerase chain reaction (PCR) using primers $g \ln B$-expr-F1 (CA TGGGATCCATGCTTCCGGGTAAAGCCGCAAGGC) and $g \ln B$-expr-R1 (CATGGAATTCGGATAGGTGAGATCGAAT GACGAGA), which contain BamHI and EcoRI restriction sites, respectively (underlined). The PCR fragments were digested with $B a m \mathrm{HI}$ and EcoRI restriction endonucleases and ligated into BamHI-EcoRI-digested pCPP30, resulting in plasmids pCPPGlnB and pCPP30GlnBP5 (Table 4), which contain $g \ln B$ or $g \ln B P 5$, respectively, that will be expressed from both the native promoter and a plasmid lac $Z$ promoter.

\section{Western blot.}

Crude extracts were prepared as described previously (Yurgel and Kahn 2008; Yurgel et al. 2010). S. meliloti anti-GSII antisera (Shatters et al. 1989) were used to visualize this protein.

\section{Plant tests.}

Alfalfa (Medicago sativa 'Champ') was used for all nodulation studies. The plant tests were performed as described previously (Yurgel et al. 2007). At 4 to 5 weeks after inoculation, plants were harvested; shoot dry mass and root nodule formation were examined.

\section{${ }^{15} \mathrm{~N}_{2}$ reduction.}

Plants were grown for 4 weeks and removed from the growth boxes, and the roots were washed. Plants roots were layered between damp papers, placed together in a 500-ml bottle to which a $10 \%$ volume of ${ }^{15} \mathrm{~N}_{2}$ (98 atom\%, Sigma-Aldrich, St. Louis) was added, and incubated for $24 \mathrm{~h}$ in a growth chamber with light. Then, the plants were separated into nodule, root, and shoot fractions and dried. Samples were ground to a fine powder and approximately $1 \mathrm{mg}$ of material was placed in a tin capsule and combusted in a Costech ECS 4010 (Valencia, CA, U.S.A.) elemental analyzer. The resulting $\mathrm{N}_{2}$ gas was injected into a Micromass (Manchester, U.K.) Isoprime isotope ratio mass spectrometer to determine ${ }^{15} \mathrm{~N} /{ }^{14} \mathrm{~N}$ ratios. Typical precision of analyses was $\pm 0.5 \%$ o for $\partial^{15} \mathrm{~N}$, where $\partial=1,000 \times\left(R_{\text {sample }} / R_{\text {standard }}\right)-1 \%$ and $R={ }^{15} \mathrm{~N} /{ }^{14} \mathrm{~N}$. The standard for $\partial^{15} \mathrm{~N}$ is atmospheric nitrogen. Higher $\partial$ values correspond to higher ${ }^{15} \mathrm{~N}$ content.

\section{ACKNOWLEDGMENTS}

This work was supported by the Agricultural Research Center at Washington State University (WSU) and grant DE-FG03-96ER20225 from the Energy Biosciences Program at the United States Department of Energy. We thank the WSU Laboratory for Biotechnology and Bioanalysis for sequencing support, R. Lee for his help with ${ }^{15} \mathrm{~N}$ analysis, D. Kahn for Rm2011 derivatives, K. Hagberg for assistance with measuring PII-UMP modifications, and M. Mortimer for assistance with ${ }^{15} \mathrm{~N}_{2}$ labeling.

\section{LITERATURE CITED}

Amar, M., Patriarca, E. J., Manco, G., Bernard, P., Riccio, A., Lamberti, A., Defez, R., and Iaccarino, M. 1994. Regulation of nitrogen metabolism is altered in a $g \ln B$ mutant strain of Rhizobium leguminosarum. Mol. Microbiol. 11:685-693.

Atkinson, M. R., and Ninfa, A. J. 1998. Role of the GlnK signal transduction protein in the regulation of nitrogen assimilation in Escherichia coli. Mol. Microbiol. 29:431-447.

Atkinson, M. R., Kamberov, E. S., Weiss, R. L., and Ninfa, A. J. 1994. Reversible uridylylation of the Escherichia coli PII signal transduction protein regulates its ability to stimulate the dephosphorylation of the transcription factor nitrogen regulator I (NRI or NtrC). J. Biol. Chem. 269:28288-28293.

Atkinson, M. R., Blauwkamp, T. A., and Ninfa A. J. 2002. Context-dependent functions of the PII and GlnK signal transduction proteins in Escherichia coli. J. Bacteriol. 184:5364-5375.

Arcondéguy, T., Huez, I., Fourment, J., and Kahn, D. 1996. Symbiotic nitrogen fixation does not require adenylylation of glutamine synthetase I in Rhizobium meliloti. FEMS (Fed. Eur. Microbiol. Soc.) Microbiol. Lett. 145:33-40.

Arcondéguy, T., Huez, I., Tillard, P., Gangneux, C., de Billy, F., Gojon, A., Truchet, G., and Kahn, D. 1997. The Rhizobium meliloti PII protein, which controls bacterial nitrogen metabolism, affects alfalfa nodule development. Genes Dev. 11:1194-1206.

Arcondéguy, T., Jack, R., and Merrick, M. 2001. $\mathrm{P}_{\mathrm{II}}$ signal transduction proteins, pivotal players in microbial nitrogen control. Microbiol. Mol. Biol. Rev. 65:80-105.

Baba, T., Ara, T., Hasegawa, M., Takai, Y., Okumura, Y., Baba, M., Datsenko, K. A., Tomita, M., Wanner, B. L., and Mori, H. 2006. Construction of Escherichia coli K-12 in-frame, single-gene knockout mutants: The Keio collection. Mol. Syst. Biol. 2:2006-2008.

Batut, J., Terzaghi, B., Gherardi, M., Huguet, M., Terzaghi, E., Garnerone, A. M., Boistard, P., and Huguet, T. 1985. Localization of a symbiotic fix region on Rhizobium meliloti pSym megaplasmid more than 200 kilobases from the nod-nif region. Mol. Gen. Genet. 1999:232-239.

Colnaghi, R., Rudnick, P., He, L., Green, A., Yan, D., Larson, E., and Kennedy, C. 2001. Lethality of $g \ln D$ null mutations in Azotobacter vinelandii is suppressible by prevention of glutamine synthetase ade- 
nylylation. Microbiology 147:1267-1276.

Colonna-Romano, S., Patriarca, E. J., Amar, M., Bernard, P., Manco, G., Lamberti, A., Iaccarino, M., and Defez, R. 1993. Uridylylation of the PII protein in Rhizobium leguminosarum. FEBS (Fed. Eur. Biochem. Soc.) Lett. 330:95-98.

Finan, T. M., Hartwieg, E., LeMieux, K., Bergman, K., Walker, G. C., and Signer E. R. 1984. General transduction in Rhizobium meliloti. J. Bacteriol. 159:120-124.

Forchhammer, K., Hedler, A., Strobel, H., and Weiss, V. 1999. Heterotrimerization of PII-like signalling proteins: Implications for PII-mediated signal transduction systems. Mol. Microbiol. 33:338-349.

Graf, J., and Ruby, E. G. 2000. Novel effects of a transposon insertion in the Vibrio fischeri glnD gene: Defects in iron uptake and symbiotic persistence in addition to nitrogen utilization. Mol. Microbiol. 37:168-179.

Huang, H. C., He, S., Bauer, Y. D. W., and Collmer, A. 1992. The Pseudomonas syringae pv. syringae $61 \mathrm{hrpH}$ product, an envelope protein required for elicitation of the hypersensitive response in plants. J. Bacteriol. 174:6878-6885.

Jiang, P., Peliska, J. A., and Ninfa A. J. 1998. Enzymological characterization of the signal-transducing uridylyltransferase/uridylyl-removing enzyme (EC 2.7.7.59) of Escherichia coli and its interaction with the PII protein. Biochemistry 37:12782-12794.

Ninfa, A. J., and Atkinson, M. R. 2000. PII signal transduction proteins. Trends Microbiol. 8:172-179.

Reitzer, L. 2003. Nitrogen assimilation and global regulation in Escherichia coli. Annu. Rev. Microbiol. 57:155-176.

Rudnick, P. A., Arcondéguy, T., Kennedy, C. K., and Kahn, D. 2001. glnD and mviN are genes of an essential operon in Sinorhizobium meliloti. J. Bacteriol. 183:2682-2685.

Sambrook, J., Fritsch, E. F., and Maniatis, T. 1989. Molecular Cloning: A Laboratory Manual, 2nd ed. Cold Spring Harbor Laboratory Press, Cold Spring Harbor, NY, U.S.A.

Schlüter. A., Nöhlen, M., Krämer, M., Defez, R., and Priefer, U. B. 2000. The Rhizobium leguminosarum bv. viciae $g \ln D$ gene, encoding a uridylyltransferase/uridylyl-removing enzyme, is expressed in the root nodule but is not essential for nitrogen fixation. Microbiology 146:29872996.

Shatters, R. G., Liu, Y., and Kahn, M. L. 1993. Isolation and characterization of a novel glutamine synthetase from Rhizobium meliloti. J. Biol. Chem. 268:469-475.

Simon, R., Priefer, U., and Pühler, A. 1983. Page 98-106 in: Molecular Genetics of the Bacterial-Plant Interaction: The Rhizobium melilotiMedicago sativa System. A. Pühler, ed. Springer-Verlag, Berlin.

Somerville, J. E., and Kahn, M. L. 1983. Cloning of the glutamine synthetase I gene from Rhizobium meliloti. J. Bacteriol. 156:168-176.

Sun, J., Van Dommelen, A., Van Impe, J., and Vanderleyden, J. 2002. Involvement of $g \ln B, g \ln Z$, and $g \ln D$ genes in the regulation of poly-3-hydroxybutyrate biosynthesis by ammonia in Azospirillum brasilense Sp7. Appl. Environ. Microbiol. 68:985-988.

Tøndervik, A., Torgersen, H. R., Botnmark, H. K., and Strøm, A.R. 2006. Transposon mutations in the $5^{\prime}$ end of $g \ln D$, the gene for a nitrogen regulatory sensor, that suppress the osmosensitive phenotype caused by otsBA lesions in Escherichia coli. J. Bacteriol. 188:4218-4226.

van Heeswijk, W. C., Wen, D., Clancy, P., Jaggi, R., Ollis, D L., Westerhoff, H. V., and Vasudevan, S. 2000. The Escherichia coli signal transducers PII $(\mathrm{GlnB})$ and $\mathrm{GlnK}$ form heterotrimers in vivo: Fine tuning the nitrogen signal cascade. Proc. Natl. Acad. Sci. U.S.A. 97:3942-3947.

Yurgel, S. N., and Kahn, M. L. 2008. A mutant GlnD nitrogen sensor protein leads to a nitrogen-fixing but ineffective Sinorhizobium meliloti symbiosis with alfalfa. Proc. Natl. Acad. Sci. U.S.A. 105:18958-18963.

Yurgel, S. N., Berrocal, J., Wilson, C., and Kahn, M. L. 2007. Pleiotropic effects of mutations that alter the Sinorhizobium meliloti cytochrome $c$ respiratory system. Microbiology 153:399-410.

Yurgel, S. N., Rice, J., Mulder, M., and Kahn, M. L. 2010. GlnB/GlnK PII proteins and regulation of the Sinorhizobium meliloti $\mathrm{Rm} 1021$ nitrogen stress response and symbiotic function. J. Bacteriol. 192:2473-2481.

Zhang, Y., Pohlmann, E. L., Serate, J., Conrad, M. C., and Roberts, G. P. 2010. Mutagenesis and functional characterization of the four domains of GlnD, a bifunctional nitrogen sensor protein. J. Bacteriol. 192:27112721. 